\title{
Dental Implants Fatigue as a Possible Failure of Implantologic Treatment: The Importance of Randomness in Fatigue Behaviour
}

\author{
María Prados-Privado, ,'2 Juan Carlos Prados-Frutos, ${ }^{1}$ Ángel Manchón, ${ }^{1}$ Rosa Rojo, \\ Pietro Felice, ${ }^{3}$ and José Antonio Bea ${ }^{4}$ \\ ${ }^{1}$ Department of Stomatology, Rey Juan Carlos University, C/ Tulipán s/n, Móstoles, 28933 Madrid, Spain \\ ${ }^{2}$ Applied Modelling and Instrumentation Group, Aragón Institute of Engineering Research, University of Zaragoza, \\ C/ Mariano Esquillor s/n, 50018 Zaragoza, Spain \\ ${ }^{3}$ Department of Biomedical and Neuromotor Sciences, Unit of Periodontology and Implantology, University of Bologna, \\ Via Zamboni 33, 40126 Bologna, Italy \\ ${ }^{4}$ Aragón Institute of Engineering Research, University of Zaragoza, C/ Mariano Esquillor s/n, 50018 Zaragoza, Spain \\ Correspondence should be addressed to María Prados-Privado; maria.prados@urjc.es
}

Received 22 June 2015; Revised 17 August 2015; Accepted 1 September 2015

Academic Editor: David M. Dohan Ehrenfest

Copyright (C) 2015 María Prados-Privado et al. This is an open access article distributed under the Creative Commons Attribution License, which permits unrestricted use, distribution, and reproduction in any medium, provided the original work is properly cited.

\begin{abstract}
Objective. To show how random variables concern fatigue behaviour by a probabilistic finite element method. Methods. Uncertainties on material properties due to the existence of defects that cause material elastic constant are not the same in the whole dental implant the dimensions of the structural element and load history have a decisive influence on the fatigue process and therefore on the life of a dental implant. In order to measure these uncertainties, we used a method based on Markoff chains, Bogdanoff and Kozin cumulative damage model, and probabilistic finite elements method. Results. The results have been obtained by conventional and probabilistic methods. Mathematical models obtained the same result regarding fatigue life; however, the probabilistic model obtained a greater mean life but with more information because of the cumulative probability function. Conclusions. The present paper introduces an improved procedure to study fatigue behaviour in order to know statistics of the fatigue life (mean and variance) and its probability of failure (fatigue life versus probability of failure).
\end{abstract}

\section{Introduction}

Fatigue phenomenon is known as the change that appears on materials when cyclic loads are applied. It is possible to find a definition in the report entitled General Principles for Fatigue Testing of Metals, which was published in 1964 by The International Organization for Standardization in Switzerland. In this report, fatigue is defined as "a term which applies to changes in properties which can occur in a metallic material due to the repeated application of stresses or strains, although usually this term applies specially to those changes with lead to cracking or failure" [1].

Dental implants have to support many loading cycles during their life; majority of those cycles are produced during physiologic masticatory cycles or parafunctions as bruxism
[2]. These additional occlusive forces produce an extra effort in the dental implant and, due to this, fatigue plays a very important role here. Masticatory forces are not constant; they change its value (direction, mean value, etc.), depending on other factors such as the constrains at the joints and bite point, the inclusion, or not, of the periodontal ligament, or the material properties assigned to the cancellous bone tissue; this involves a lot of randomness associated with the fatigue phenomenon. To reduce the unrestrained elements, we have used a method to show how uncertainties concern fatigue life and the probability of failure using a method based on Markoff chains, Bogdanoff and Kozin cumulative damage model, and probabilistic finite elements method [3-5]. 


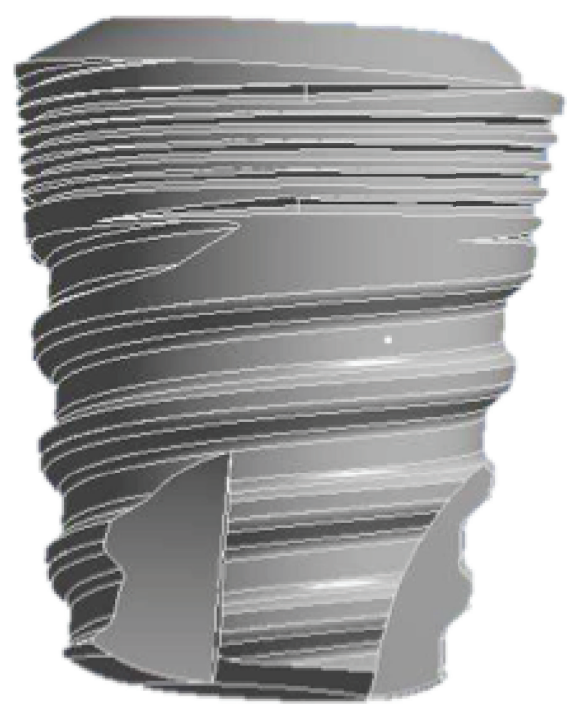

FIGURE 1: Proclinic dental implant analysed.

Among the factors described as possibly responsible for the failure of implant treatment due to mechanical etiology, it is the clinical phenomenon known as bruxism; the American Academy of Orofacial Pain (AAOP, 2008) defines it such as in [6] "as a movement disorder of the stomatognathic system characterized." The grinding and clenching of teeth, either day or night, are with a prevalence between 6 and $91 \%$ for both sexes in the general population [7], and the age range is between 18 and 49 years [8]. The clinical consequences of bruxism may be different for its different types (awake and sleep), highlighting muscular hypertrophy, tooth wear, tooth fracture, fracture of the restorations or of the implants, sensitivity or pain of the teeth, muscle or joints, and temporomandibular joint disc displacement [7].

Bruxism causes excessive load on dental implants and their superstructures, causing bone loss around implants or even failure of the implant and/or of the implant restoration. Therefore, bruxism is considered a risk factor for implant treatment [9-12].

In order to evaluate the dental implant design propriety, it is necessary to carry out standard fatigue tests with different loads levels, as is explained in ISO 14801. These tests require a lot of time to do all the analysis and high expensive resources because in the practical way to study fatigue it is necessary to analyse a great deal of dental implants until their fracture. The aim of this study was to evaluate the predicted fatigue life of Proclinic dental implant using Markoff chains, Bogdanoff and Kozin cumulative damage model, and the probabilistic finite element method to reduce time and improve dental implants and compare the results with some mathematical models to study fatigue, for example, Goodman or Gerber models [13].

\section{Materials and Methods}

In this paper we have analysed a Proclinic dental implant called CON.INT IP887 (Figure 1) which has a hexagonal internal connection, a diameter $D=5 \mathrm{~mm}$, and a length
$L=6 \mathrm{~mm}$. The IGES file used in this study has been provided by Proclinic dental implants, manufactured by Avenir S.L. (Rimini, Italy).

Proclinic dental implant that we have analysed is manufactured of a titanium alloy known as Titanium ELI (Ti6Al4V), with an internal hexagonal connection and with characteristics shown below. This alloy shows excellent performance in vivo due to the excellent balance between mechanical, physicochemical, and biofunctional properties [14].

Titanium is a highly biocompatible biomaterial (both in vitro and in vivo), also bioinert, with a great ability to establish a direct structural and functional connection between ordered and living bone and the surface of the implant [15].

Wrought Ti-6A1-4V is a useful material for surgical implants because of its low modulus, good tensile and fatigue strength, and biological compatibility. It is used for bone screws and for partial and total hip, knee, elbow, jaw, finger, and shoulder replacement joints. Where fatigue properties are not an issue, the cast alloy also has had minor use as an implant product [16].

Geometry in IGES format has been used to generate the finite element mesh employing the software ANSYS CFX (version 14.5, Canonsburg, Pennsylvania, United States). 25427 nodes and 14481 elements compose this mesh. The boundary conditions applied match ISO 14801 test procedure, that is,

(i) Apical: all DOFs (degrees of freedom, i.e., displacements) restrained,

(ii) Thread: only displacements normal to the surface are allowed,

(iii) Crestal: natural boundary conditions, that is, force, are applied with an orientation of 15 degrees as described.

2.1. Conventional Fatigue by Mathematical Models. In this section, we show a quick review about the three more important models in order to remind the reader about those common criteria and, in this way, compare them with the method proposed in this study. Fatigue behaviour is usually studied theoretically by three mathematical models, which were proposed by Goodman, Gerber [13], and Soderberg [17].

These three relationships, as Meyers and Chawla explain in [18], consider the cyclic load as appears in Figure $2, \sigma_{m}$ being the mean stress and $\sigma_{a}$ being the fatigue strength in terms of stress amplitude when $\sigma_{m}=0$ (stress amplitude).

Mathematically, these three models are expressed as

$$
\begin{aligned}
\text { Goodman: } \sigma_{a} & =\sigma_{e}\left[1-\left(\frac{\sigma_{m}}{\sigma_{u}}\right)\right], \\
\text { Gerber: } \sigma_{a} & =\sigma_{e}\left[1-\left(\frac{\sigma_{m}}{\sigma_{u}}\right)^{2}\right], \\
\text { Soderberg: } \sigma_{a} & =\sigma_{e}\left[1-\left(\frac{\sigma_{m}}{\sigma_{y}}\right)\right],
\end{aligned}
$$




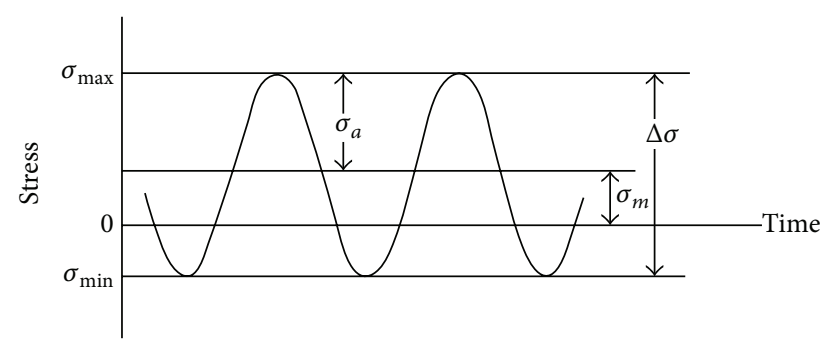

Figure 2: Cyclic loads.

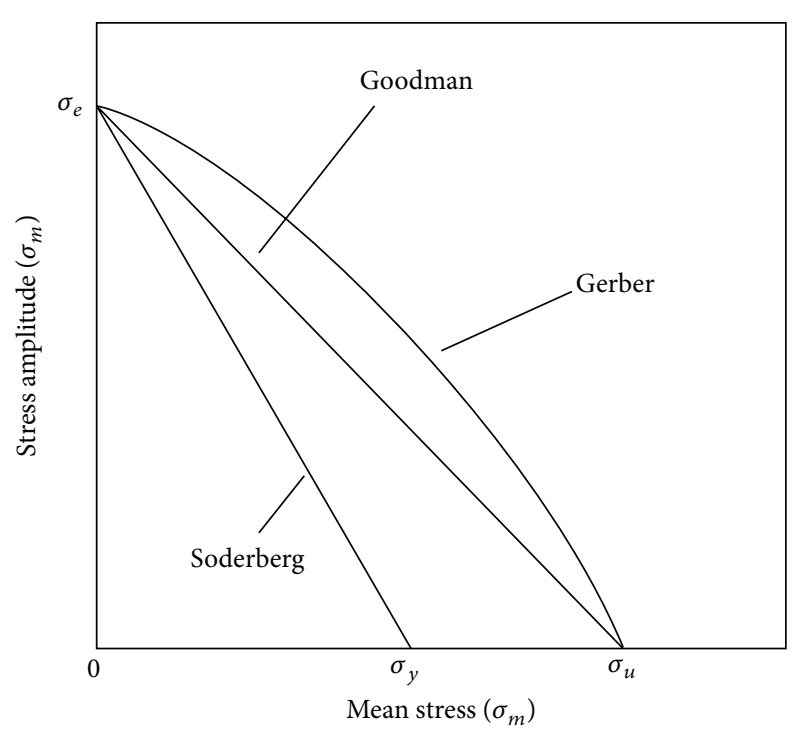

Figure 3: Goodman, Soderberg, and Gerber graphical models.

where $\sigma_{u}$ is the ultimate tensile strength and $\sigma_{y}$ is the yield strength. Those three expressions can be represented graphically as ASM shows (Figure 3) [14].

The general trend given by the Goodman relation is one of decreasing fatigue life with increasing mean stress for a given level of applied stress. The relation can be plotted to determine the safe cyclic loading of a part; if the coordinate given by the mean stress and the applied stress lies under the curve given by the relation, then the part will survive. If the coordinate is above the curve, then the part will fail for the given stress parameters.

Gerber used a parabolic model and Goodman used a line, which are more conservative [19].

2.2. Probabilistic Fatigue Method. Proposal presented here is based on the following questions:

(1) Why is it necessary to do a lot of tests with the use, probably excessive, of time and components, uniquely dental implants in this case, and what that implies?

(2) What happens to random variables that models discussed above do not consider? Models proposed by Soderberg, Gerber, and Goodman do not take into account that both masticatory forces and material

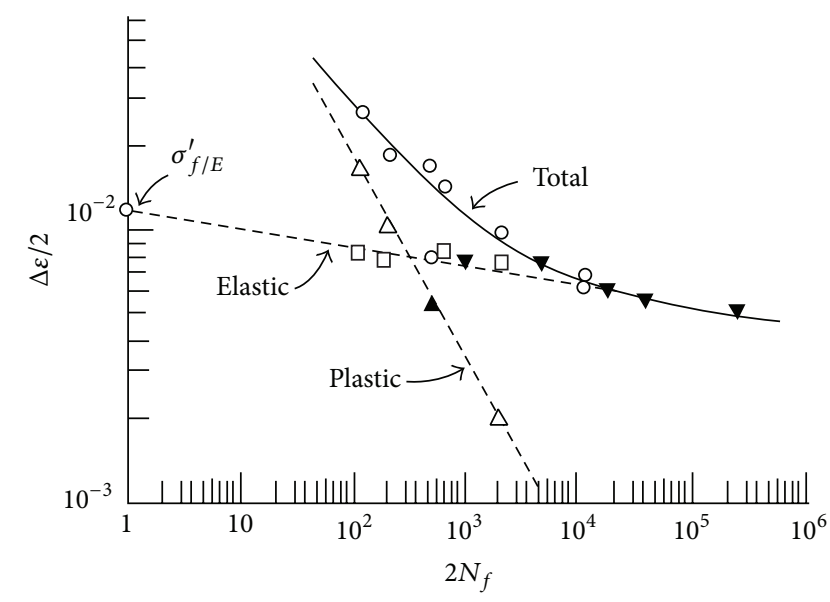

FIgURE 4: $\varepsilon-N$ curve.

properties do not always have the same value, as can be appreciated in (1).

Most of the fatigue studies are done from the deterministic point of view, while the model used in this study considers the randomness of some variables. The most important difference between the three models explained below and the model used in this work is that in the last one it is possible to take into account defects that can appear in the dental implant and the different mean loads that vary depending on the patient and the masticatory loads.

In order to determine the fatigue life with random variables and to be able to predict the probability of failure for each cycle, authors employed a probabilistic model developed by Bogdanoff and Kozin (B-K), which is based on Markoff chains. To generate the model, we have used the results taken from constant load structural analysis (von Mises stress) employing the commercial software ANSYS CFX.

According to the proposal by Prados-Privado et al. [20] which combines the finite element method and the B-K model it is possible to solve the problem in four steps (one associated with the deterministic problem and three associated with the random variables considered).

The main ideas are as follows:

(i) Once the mean and the variance of the fatigue life are known, we are ready to construct the probability transition matrix (PTM). Equations (3) and (4) from the Supplementary Material (available online at http://dx.doi.org/10.1155/2015/825402) allow calculating the parameters needed to construct the PTM.

(ii) To get the mean life, it is necessary to know the $\varepsilon-N$ curve of the material. Figure 4 shows an example of how $\varepsilon-N$ curve is.

(iii) To get the variance of the fatigue life, it is necessary to know some elastoplastic properties of the material (Neuber's law). In Figure 5 is represented the graphical formulation for Neuber's law, which has been obtain in [21]. 


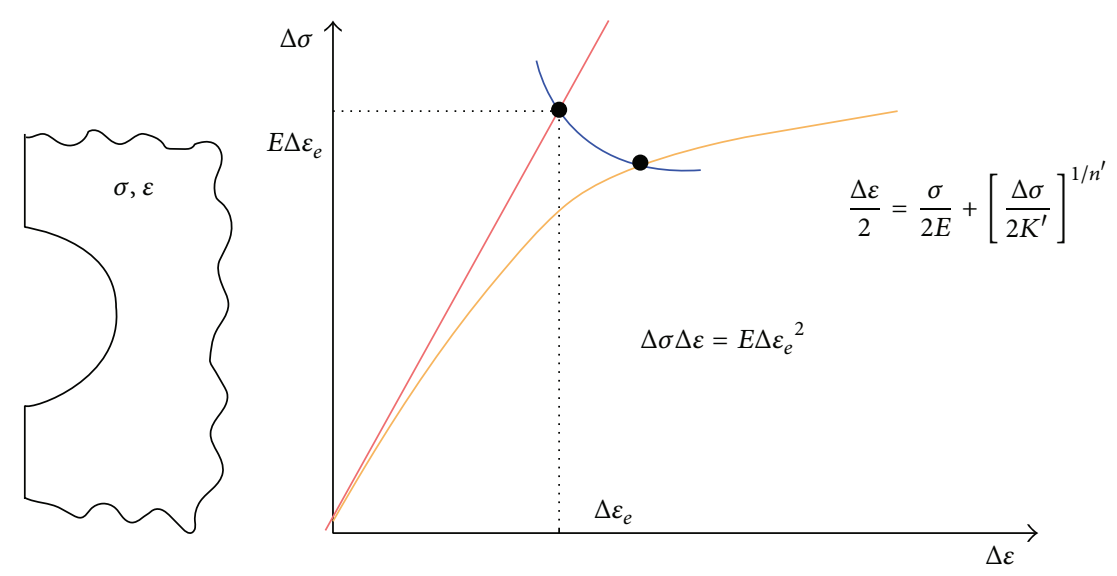

FIgURE 5: Graphical formulation of Neuber's law.

(iv) To get the variance of the elastic properties it is necessary to have, first, a stochastic analysis, which is done by ANSYS CFX.

With the idea of a good understanding of the results, here we include a brief description of the probability transition matrix (PTM), as Bogdanoff and Kozin wrote in [3], which has the form shown in the following expression:

$$
\mathbf{P}=\left(\begin{array}{cccccc}
p_{1} & q_{1} & 0 & \cdots & 0 & 0 \\
0 & p_{2} & q_{2} & 0 & \cdots & 0 \\
0 & 0 & p_{3} & q_{3} & \cdots & 0 \\
\vdots & \vdots & \vdots & \vdots & \ddots & \vdots \\
0 & 0 & 0 & \cdots & p_{b-1} & q_{b-1} \\
0 & 0 & 0 & \cdots & 0 & 1
\end{array}\right),
$$

where $p_{j}$ is the probability of remaining in the same stage $j$ during damage cycle and $q_{j}$ is the probability of jumping to the next level that is from the damage state $j$ to $j+1$. Obviously, $p_{j}+q_{j}=1$ and $0<p_{j}<1$. Parameters and expressions required to construct this matrix are included in the Supplementary Material.

This matrix is very important in this study because, without it, it is not possible to calculate our principal aim; in other words, with this matrix we can calculate the dental implant probability of failure.

To obtain the PTM, it is necessary to compute statistics (mean value and variance) of the fatigue life. Bogdanoff and Kozin did it directly from experimental data. The first time that these statistics were numerically evaluated was done by Bea et al. [4]. A choice could be to obtain samples from Monte Carlo Simulation, which is really expensive (it is necessary to compute a hundred or thousand times of fatigue life simulations). We have chosen the PFEM (perturbation method). Instead of generating samples, several Taylor expansions are done around every random variable that affects fatigue life, for all the random fields in continuum mechanics: displacement field, strain field, and stress field. If these fields are known, the fatigue life can be computed $[4,20,21]$.
More information about the method used in this paper can be found in the Supplementary Material.

\section{Results}

The main aim of this section is to show the difference between the conventional way and the method we propose here to study fatigue behaviour. In this case, we are applying the method on Proclinic dental implant called CON.INT IP887 shown in Figure 1 using the commercial software Mathematica (version 9, Oxfordshire, United Kingdom).

3.1. Material Properties. Table 1 shows the material properties used in this study. These values have been proportioned by Proclinic and obtained from ASM and Kobayashi et al. [22].

3.2. Force Bite. In this study, we have used mean and standard deviation on bite force shown in Table 2 and expressed in Newton (SI force units). These values have been obtained as a mean on values that Clark and Carter used in their study [23]. Values in Table 2 are a statistical analysis about the bite force in both genders without any health and dentist problems done with their molars.

3.3. Analysis. The most important results gained by employing the probabilistic model are shown in Table 3. From a conventional way, the same result is obtained. This means that, in this case, this implant should have the same life independently of the method used. The probabilistic model proposed here gets a bigger life but, however, it is more precise because this model provides the mean fatigue life, the variance for this life, and a cumulative probability function as Figure 7 shows.

It should be pointed out that there is no variance on the three first models because they are deterministic; because of that variance is only applicable in the probabilistic model.

Figure 6 shows the life range in which the Proclinic dental implant analysed is going to be.

Here we find the first main difference between the results obtained by conventional fatigue and the probabilistic model 
TABLE 1: Titanium alloy properties employed.

\begin{tabular}{lccccccc}
\hline Emblem & $E[\mathrm{GPa}]$ & $b[-]$ & $c[-]$ & $\sigma_{f^{\prime}}[\mathrm{Pa}]$ & $\varepsilon_{f^{\prime}}[-]$ & $\sigma_{y}[\mathrm{MPa}]$ & $\sigma_{u}[\mathrm{MPa}]$ \\
\hline Value & 114 & $-0,018$ & $-0,026$ & $1,4 \cdot 10^{9}$ & 0,0186 & 828 & 895 \\
\hline
\end{tabular}

$E$ : modulus of elasticity (whose units are Pascal, SI pressure units).

$b$ : fatigue resistance exponent (nondimensional).

$c$ : fatigue ductility exponent (nondimensional).

$\sigma_{f^{\prime}}$ : fatigue resistance coefficient $(\mathrm{Pa})$.

$\varepsilon_{f^{\prime}}$ : fatigue ductility coefficient (nondimensional).

$\sigma_{y}$ : tensile yield strength $(\mathrm{Pa})$.

$\sigma_{u}$ : tensile ultimate strength $(\mathrm{Pa})$.

TABLE 2: Bite force: mean and standard deviation.

\begin{tabular}{lc}
\hline Mean $[N]$ & Standard deviation $[N]$ \\
\hline 583,49 & 72,6 \\
\hline
\end{tabular}

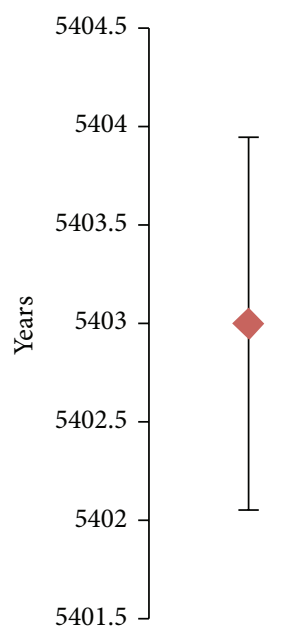

FIgURE 6: Minimum and maximum life.

proposed in this paper. The results show a big difference, but it is necessary to understand that the result gained by conventional fatigue means that the fatigue life is going to be bigger than 48 years but it does not say anything about the probability of failure.

Values obtained to get Figure 7 are those shown in Table 4. In order to get a good graph to show with detail what happens on implants, it was necessary to change the temporal axes, so, column "time" is the value which has to be multiplied by the number of cycles to know the probability of failure associated with each cycle.

Figure 7 has the following meaning:

(1) Cumulative probability function relates probability of failure with number of cycles.

(2) Until time 9, the probability of failure is zero, so, that means that nothing is waiting to occur until this time.

(3) If we get the probability of failure associated with the mean life obtained and shown in Table 3, we discover that it is very close to $50 \%$.

(4) Minimum life obtained with the method proposed here has a zero probability of failure.

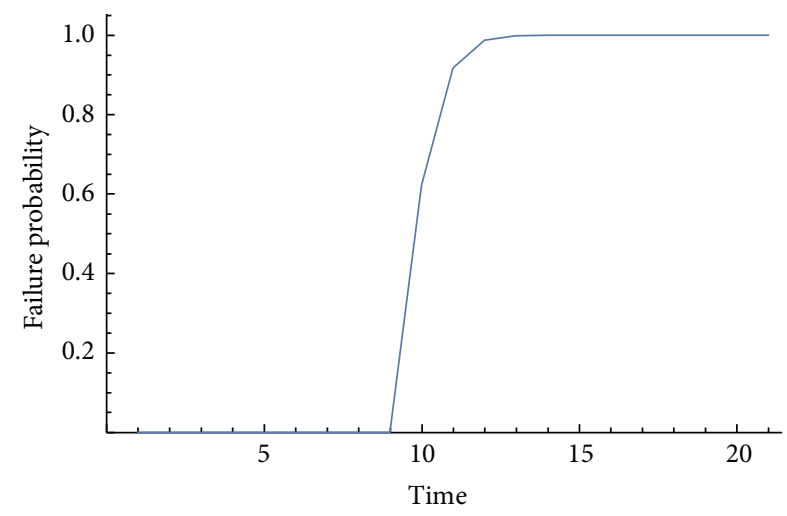

FIGURE 7: Cumulative probability function.

(5) Maximum life obtained has a $60 \%$ of probability of failure associated, approximately.

\section{Discussion and Conclusion}

In this paper we present the application of a probabilistic methodology for dental implants, taking into account the variability in loads, fatigue behaviour, and design aspects and comparing results with deterministic fatigue. This probabilistic methodology, based on Markoff chains, was first applied by Bea et al. [4,21], to metal fatigue including separately crack nucleation and crack growth stage.

Uncertainties between mastication habits among different patients imply that random loads are applied to the implants and since this must be taken into account in the model from the very beginning it can be done using Markoff chains. Most FE studies on dental implants are static analyses [24-29].

This methodology allows us to keep in mind different load blocks with different amplitudes, considering their sequential effects. These sequential effects achieve more realistic and confident results and they are not used in this study because the aim is to present the main difference between the conventional way of studying fatigue behaviour and a probabilistic method.

Main differences between the conventional way and the method we have proposed here are as follows:

(i) First of all, conventional fatigue studies do not take into account uncertainness that material or loads can have. 
TABLE 3: Fatigue results for Proclinic dental implant analysed.

\begin{tabular}{lcccc}
\hline & Goodman & Soderberg & Gerber & Probabilistic model \\
\hline \multirow{2}{*}{ Life } & $>1,7 \cdot 10^{9}$ cycles & $>1,7 \cdot 10^{9}$ cycles & $>1,7 \cdot 10^{9}$ cycles & $1,18 \cdot 10^{11}$ cycles \\
& $(>48,7$ years $)$ & $(>48,7$ years $)$ & $(>48,7$ years $)$ & $(5403$ years $)$ \\
\hline \multirow{2}{*}{ Variance } & & Not applicable & $4,28 \cdot 10^{14}$ \\
& & & $\left(0.89\right.$ years $\left.^{2}\right)$ \\
\hline
\end{tabular}

TABLE 4: PTM parameters.

\begin{tabular}{lccc}
\hline Matrix dimension & Time & $p$ & $q$ \\
\hline 3500 & 1.54 & 0.000106675 & 0.999893 \\
\hline
\end{tabular}

(ii) With the probabilistic model, it is possible to know the mean fatigue life, the variance, and, what is more important, the probability of failure for each cycle.

(iii) In this case, results are the same for the three models used to study conventional fatigue life.

(iv) As Figure 7 shows, it is possible to ensure that the minimum fatigue life for this dental implant is 5388 years, because, below this time, the probability of failure is zero.

(v) There is a big difference in fatigue life obtained by deterministic or probabilistic models. This means that with the probabilistic method we are able to know with more detail the behaviour that Proclinic dental implant analysed here is going to have, keeping in mind always that this case is also including random variables, which introduced more confidence in the model and results obtained.

\section{Abbreviations \\ ASM: American Society of Metals \\ B-K: Bogdanoff and Kozin \\ PTM: Probability transition matrix \\ FE: Finite element.}

\section{Conflict of Interests}

The authors declare that there is no conflict of interests regarding the publication of this paper.

\section{Authors' Contribution}

María Prados-Privado, Juan Carlos Prados-Frutos, and José Antonio Bea contributed equally to this paper.

\section{Acknowledgments}

Proclinic-URJC Grant A-285 financially supported the research for this paper.

\section{References}

[1] S. Suresh, Fatigue of Materials, Cambridge University Press, New York, NY, USA, 2006.

[2] J. M. Ayllón, C. Navarro, J. Vázquez, and J. Domínguez, "Fatigue life estimation in dental implants," Engineering Fracture Mechanics, vol. 123, pp. 34-43, 2014.

[3] J. L. Bogdanoff and F. Kozin, Probabilistic Models of Cumulative Damage, Wiley-Interscience, New York, NY, USA, 1985.

[4] J. A. Bea, M. Doblaré, and L. Gracia, "Evaluation of the probability distribution of crack propagation life in metal fatigue by means of probabilistic finite element method and B-models," Engineering Fracture Mechanics, vol. 63, no. 6, pp. 675-711, 1999.

[5] R. De Leeuw, Orofacial Pain: Guidelines for Assessment, Diagnosis and Management, Quintessence Books, Chicago, Ill, USA, 2008.

[6] T. Hisada and S. Nakagiri, "Stochastic finite element method developed for structural safety and reliability," in Proceedings of the 3rd International Conference on Structural Safety and Reliability (ICOSSAR '81), pp. 395-408, Elsevier, Tronheim, Norway, December 1981.

[7] F. Lobbezoo, J. Ahlberg, D. Manfredini, and E. Winocur, "Are bruxism and the bite causally related?" Journal of Oral Rehabilitation, vol. 39, no. 7, pp. 489-501, 2012.

[8] T. Strausz, J. Ahlberg, F. Lobbezoo et al., "Awareness of tooth grinding and clenching from adolescence to young adulthood: a nine-year follow-up," Journal of Oral Rehabilitation, vol. 37, no. 7, pp. 497-500, 2010.

[9] F. Lobbezoo, J. Van Der Zaag, and M. Naeije, "Bruxism: its multiple causes and its effects on dental implants-an updated review," Journal of Oral Rehabilitation, vol. 33, no. 4, pp. 293300, 2006.

[10] F. Lobbezoo, J. E. I. G. Brouwers, M. S. Cune, and M. Naeije, "Dental implants in patients with bruxing habits," Journal of Oral Rehabilitation, vol. 33, no. 2, pp. 152-159, 2006.

[11] K. Koyano, Y. Tsukiyama, R. Ichiki, and T. Kuwata, "Assessment of bruxism in the clinic," Journal of Oral Rehabilitation, vol. 35, no. 7, pp. 495-508, 2008.

[12] A. Wennerberg and T. Albrektsson, "Current challenges in successful rehabilitation with oral implants," Journal of Oral Rehabilitation, vol. 38, no. 4, pp. 286-294, 2011.

[13] F. C. Campbell, Elements of Metallurgy and Engineering Alloys, ASM International, Novelty, Ohio, USA, 2008.

[14] S. V. Dorozhkin, "Bioceramics of calcium orthophosphates," Biomaterials, vol. 31, no. 7, pp. 1465-1485, 2010.

[15] T. Albrektsson, P.-I. Brånemark, H.-A. Hansson, and J. Lindström, "Osseointegrated titanium implants. Requirements for ensuring a long-lasting, direct bone-to-implant anchorage in man," Acta Orthopaedica Scandinavica, vol. 52, no. 2, pp. 155170, 1981.

[16] G. Welsch, R. Boyer, and E. W. Collings, Materials Properties Handbook: Titanium Alloys, ASM International, 1993. 
[17] C. R. Soderberg, "Working stresses," Journal of Applied Mechanics, vol. 57, p. 106, 1935.

[18] M. A. Meyers and K. K. Chawla, Mechanical Behavior of Materials, Cambridge University Press, New York, NY, USA, 2009.

[19] W. Gerber, "Bestimmung der zulossigen spannungen in eisenconstructionen," Z. Bayer Arch. Ing. Ver, vol. 6, 1874.

[20] M. Prados-Privado, J. C. Prados-Frutos, and J. A. Bea, "Fatigue in dental implants studied with stochastic finite element formulation and cumulative damage model," Anales de Mecánica de la Fractura, vol. 2, no. 1, pp. 315-319, 2013.

[21] J. L. Nuñez, Metal fatigue analysis on nucleation step using damage models and probabilistic finite elements [Ph.D. thesis], 2003, (Spanish).

[22] E. Kobayashi, H. Mochizuki, H. Doi, T. Yoneyama, and T. Hanawa, "Fatigue life prediction of biomedical titanium alloys under tensile/torsional stress," Materials Transactions, vol. 47, no. 7, pp. 1826-1831, 2006.

[23] G. T. Clark and M. C. Carter, "Electromyographic study of human jaw-closing muscle endurance, fatigue and recovery at various isometric force levels," Archives of Oral Biology, vol. 30, no. 7, pp. 563-569, 1985.

[24] W. K. Liu, T. B. Belytschko, and G. H. Besterfield, "Probabilistic finite element method," in Computational Mechanics of Probabilistic and Reliability Analysis, Elmepress International, Lausanne, Switzerland, 1989.

[25] L. Baggi, I. Cappelloni, F. Maceri, and G. Vairo, "Stress-based performance evaluation of osseointegrated dental implants by finite-element simulation," Simulation Modelling Practice and Theory, vol. 16, no. 8, pp. 971-987, 2008.

[26] M. A. Pérez, J. C. Prados-Frutos, J. A. Bea, and M. Doblaré, "Stress transfer properties of different commercial dental implants: a finite element study," Computer Methods in Biomechanics and Biomedical Engineering, vol. 15, no. 3, pp. 263-273, 2012.

[27] T. J. R. Hughes, Linear Static and Dynamic Finite Element Analysis, Prentice Hall, 1987.

[28] C. S. Petrie and J. L. Williams, "Comparative evaluation of implant designs: influence of diameter, length, and taper on strains in the alveolar crest-a three-dimensional finite-element analysis," Clinical Oral Implants Research, vol. 16, no. 4, pp. 486494, 2005.

[29] H. Van Oosterwyck, J. Duyck, J. V. Sloten et al., “The influence of bone mechanical properties and implant fixation upon bone loading around oral implants," Clinical Oral Implants Research, vol. 9, no. 6, pp. 407-418, 1998. 

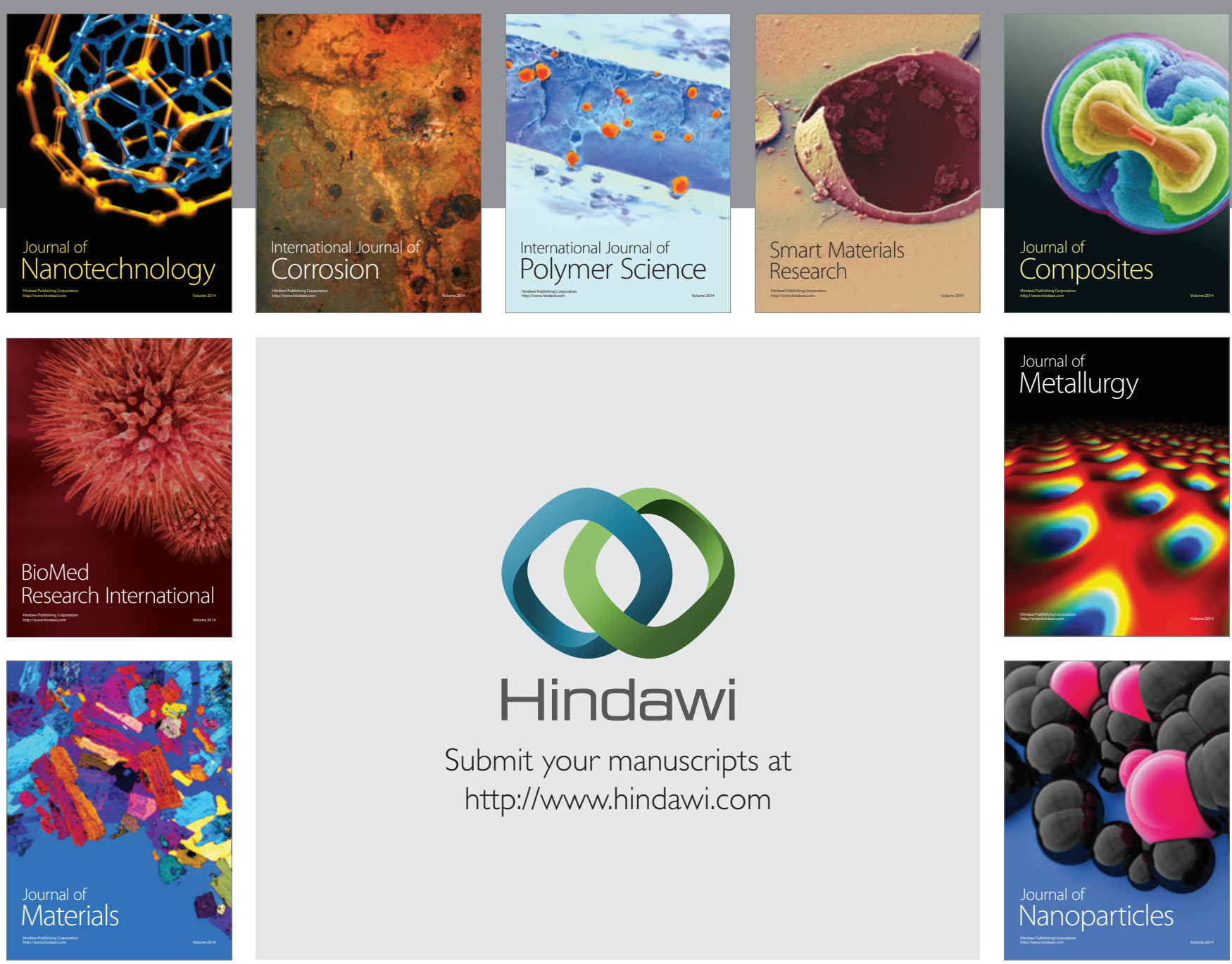

Submit your manuscripts at http://www.hindawi.com
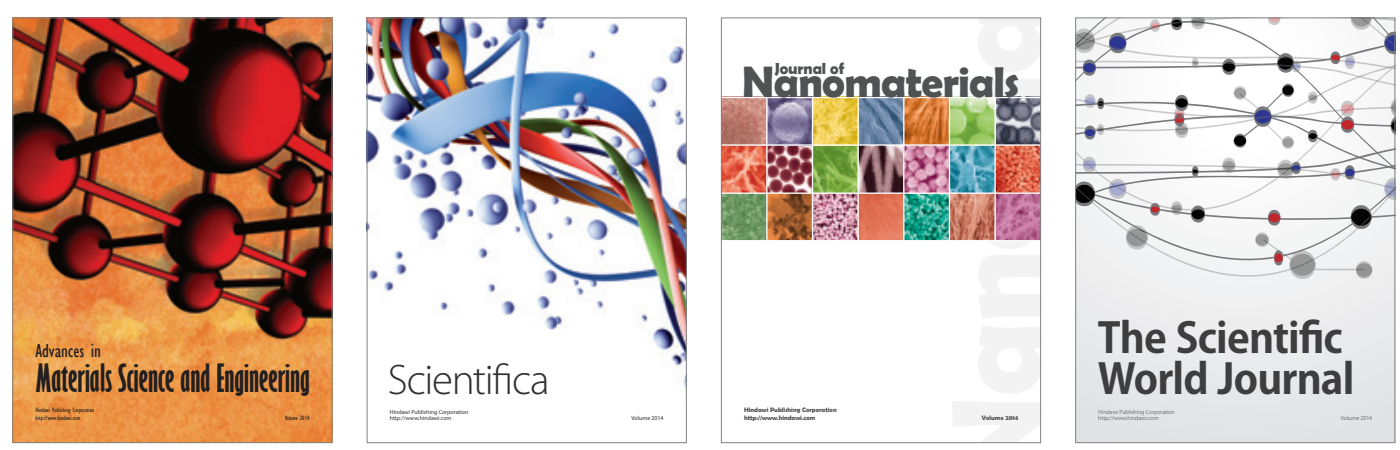

\section{The Scientific World Journal}
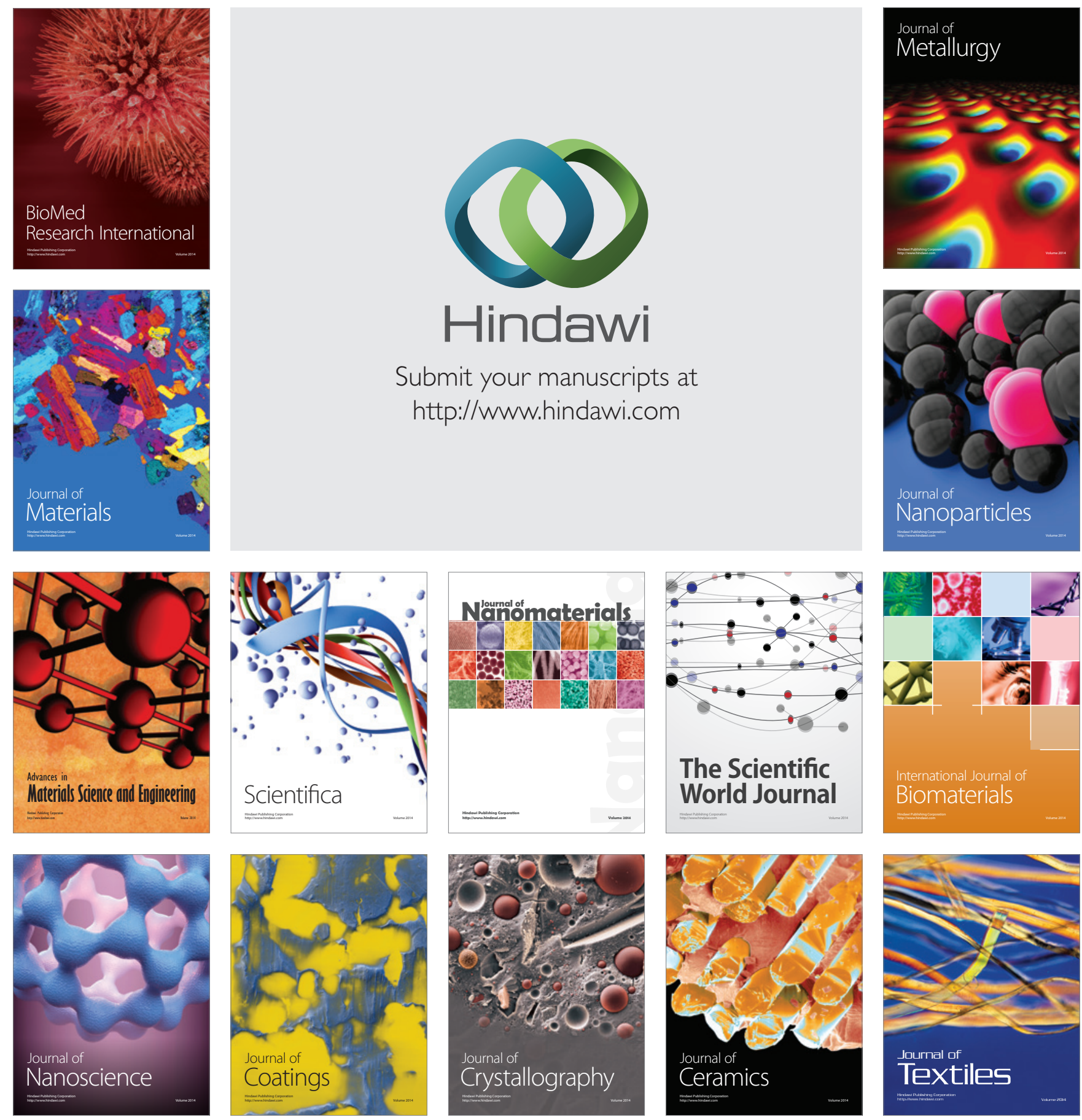